\section{Low serum haptoglobin and blood films suggest intravascular hemolysis contributes to severe anemia in hereditary hemorrhagic telangiectasia}

Hereditary hemorrhagic telangiectasia (HHT) results in iron deficiency anemia due to chronic blood losses from telangiectasia and arteriovenous malformations in the nose and gastrointestinal tract. Patients with HHT can require very frequent intravenous iron infusions or blood transfusions in addition to interventional and medical hemorrhage-limitation strategies such as bevacizumab or thalidomide. Generally, iron requirements reflect the rate of blood loss, with patients demonstrating appropriate suppression of hepcidin when iron deficient, and correcting anemia if hemorrhage reduces and iron intake is supplemented. However, clinic audits showed that for a population with presumed iron deficiency, surprisingly high proportions had non-microcytic anemias and/or were failing to correct hemoglobin in the setting of apparently sufficient iron as judged by serum ferritin. We hypothesised that reduced intravascular erythrocyte survival (hemolysis) might be augmenting anemia and introduced serum haptoglobin as a first-line screen for HHT patients where iron requirements were considered potentially excessive for evident hemorrhage. Seven of the 27 selected patients had a haptoglobin below the normal range suggesting intravascular hemolysis could be contributing to erythrocyte losses. In support, blood films demonstrated features of hemolysis (such as polychromasia and spherocytes/elliptocytes) as commonly as those of iron deficiency; patients with lower haptoglobin were more severely anemic; erythrocytes from patients with subnormal haptoglobin did not demonstrate microcytosis or hypochromia as would be expected for purely iron deficiency anemia; and several patients had HHT-independent hemolytic risks factors in addition to the turbulent blood flow through HHT arteriovenous malformations. Finally, in one transfusion-dependent patient with unexplained subnormal haptoglobins, the hemoglobin spontaneously rose by $34 \mathrm{~g} / \mathrm{L}$ after 7 days prednisolone treatment for asthma. We concluded that while the main HHT therapeutic emphasis must remain hemorrhage limitation and treatment of proven iron deficiency, intravascular hemolysis should be considered a potential contributor to anemia in a proportion of HHT patients with severe anemia. Erythrocyte protection strategies may then prove helpful adjuncts to anti-hemorrhagic and iron replacement therapies.

In detail, severe anemia as a result of iron deficiency is a common consequence of hereditary hemorrhagic telangiectasia (HHT). Almost all patients experience recurrent nosebleeds that frequently occur daily. ${ }^{1}$ A smaller proportion have chronic gastrointestinal bleeding. ${ }^{2}$ Controlling HHT blood losses reduces iron/transfusional requirements, as evidenced following nasal closure for patients with very severe epistaxis. ${ }^{3}$ Unfortunately, the majority of HHT patients have ongoing recurrent hemorrhage and, for many, this results in substantial blood losses despite multiple interventional procedures and/or medical treatments such as tranexamic acid, thalidomide, tamoxifen, and bevacizumab. Bleeding and anemia are established priorities for the HHT patient/professional consortium of the European Reference Network on Rare Multisystemic Vascular Diseases (VASCERN) - management of nosebleeds and assessment of iron deficiency are two of the five VASCERN HHT Outcome Measures. ${ }^{4}$

HHT patients are particularly heavy users of iron treatments and blood transfusions, despite normal iron handling as evaluated by appropriate suppression of hepcidin when iron deficient. ${ }^{5}$ Furthermore, as in the general population, ${ }^{6}$ serum iron and transferrin saturation index (TfSI) can increase acutely for several hours as secondary
A

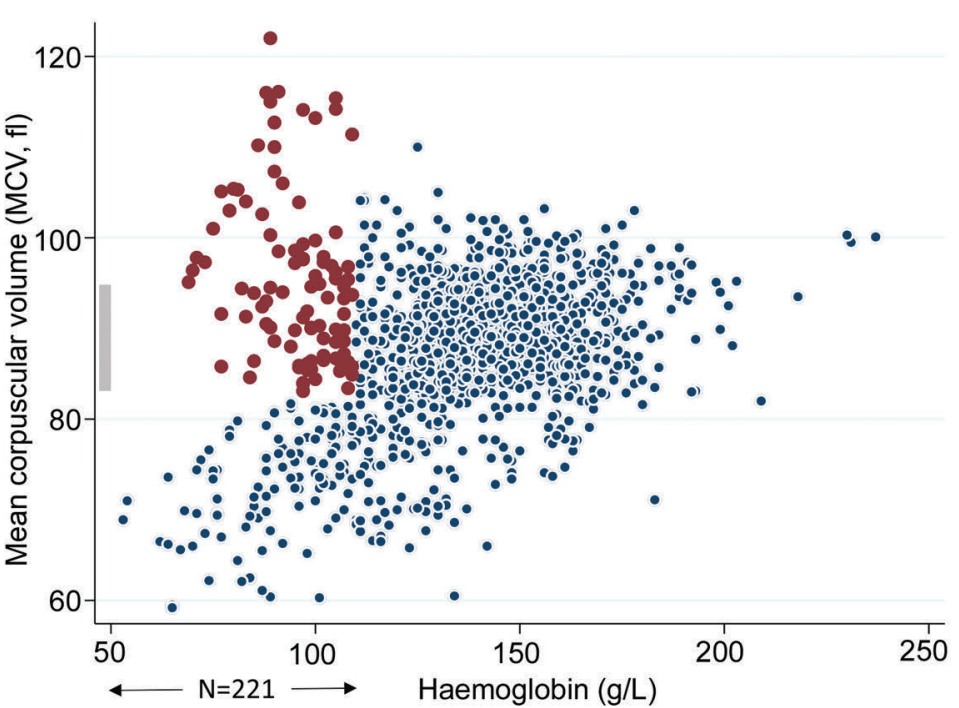

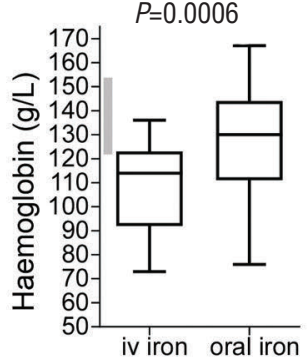

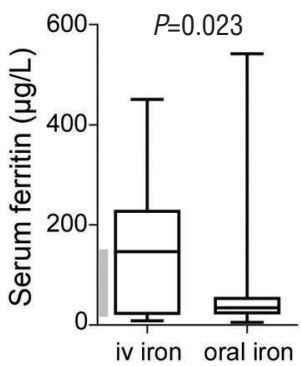

Figure 1. Non iron-deficient red cell and iron indices in HHT patients. (A) Mean corpuscular volume (MCV) in 1409 datasets of 486 HHT patients, by hemoglobin. Red values indicate normal to high values in the 221 datasets (where hemoglobin $<110 \mathrm{~g} / \mathrm{L}$ ). Note the number of datasets ranged from $1-37$ (median 3 ) per patient. (B) Anemia and iron indices in 86 consecutively reviewed HHT patients using iron- data indicate a single dataset per patient. All iron users were categorised by whether or not they used intravenous (iv) iron, or purely oral iron tablets. Upper graph: hemoglobin, lower graph: ferritin. Boxplot boxes indicate median (line) and interquartile range, error bars represent two standard deviations. Normal ranges indicated by grey bars. 
A

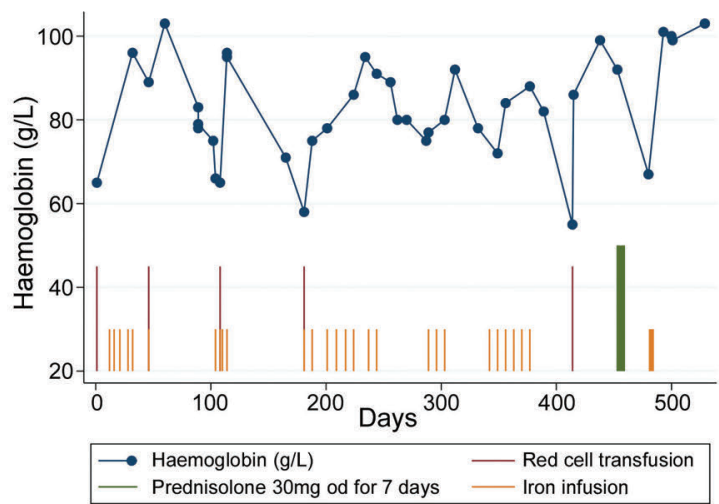

C

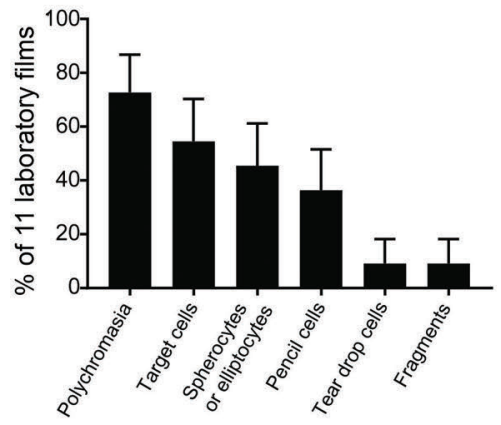

B

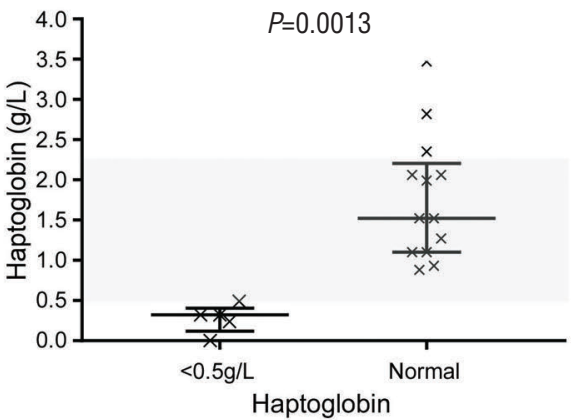

$P=0.0070$

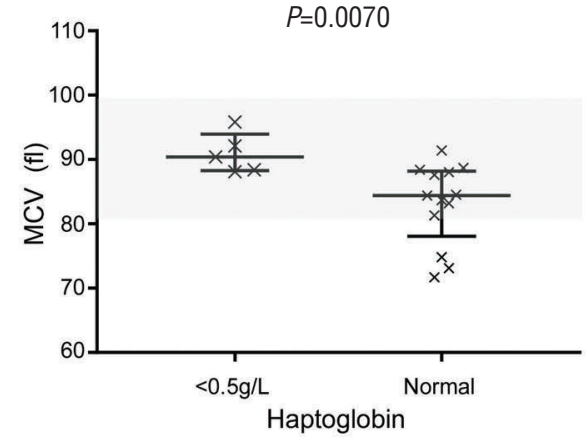

Figure 2. Hemolytic markers in HHT patients. (A) Serial measurements over 17 months of a typical severely anemic HHT patient in the cohort of 27 . Hemoglobin (blue symbols) in relation to iron infusions (yellow lines), red cell transfusions (red lines), and one-week course of prednisolone 30mg od (for asthma, green). Three serum haptoglobin measurements were below the normal range (Online Supplementary Figure S1). (B) Serum haptoglobin in 27 selected HHT patients, categorised by values above and below the lower limit of normal. Error bars demonstrate median and interquartile range. Shaded area represents normal range. C) Manual blood films reported in 11 of the 27 selected HHT patients: symbols demonstrate mean and 95\% confidence intervals. D) Mean corpuscular volume (MCV) in HHT patients without recent transfusions, categorised by haptoglobin values above and below the lower limit of normal. Symbols demonstrate median and interquartile range. Note in B-D, data represent a single dataset per patient, and D presents data from the subset of those in Figure $2 B$ who had not received a blood transfusion in the previous month. Data not shown for one patient who had previously been treated for a bone marrow pathology (though inclusion did not materially alter the analyses).

consequences of iron treatments for iron deficiency. ${ }^{7}$ But two groups of HHT patients were puzzling and led us to seek evidence for additional causes of anemia: the firstwere patients transferred from oral to intravenous iron, then to increasing frequencies of intravenous iron, while failing to correct the anemia. The second reported bleeding had reduced but high transfusion requirements continued.

Formal hemorrhage adjusted iron requirements (HAIR) were developed and evaluated, ${ }^{5}$ identifying a subgroup of HHT patients who had iron requirements out-of-proportion to known nasal, gastrointestinal, menstrual and other discernible blood losses. High proportions of clearly anemic HHT patients (hemoglobin $<110 \mathrm{~g} / \mathrm{L}$ ) did not have the expected microcytosis or hypochromia for iron deficiency $^{8}$ : for example, 106/221 (48\%) had mean corpuscular volume (MCV) measurements at or above the lower limit of normal (Figure 1A). Furthermore, of all 87 HHT patients who were using iron treatments when reviewed between June 2015 and June 2017, as a group, compared to oral iron users, intravenous iron users seemed to be failing to correct hemoglobin in the setting of apparently sufficient iron as judged by serum ferritin (Figure 1B).

Iron requirements out-of-proportion to blood losses, and two particular HHT patients (heterozygous for $\mathrm{Hb}-\mathrm{S}$ and $\mathrm{Hb}$ D-Punjab respectively), led CLS to hypothesise that reduced intravascular erythrocyte survival may be contributing to the "failure to correct anemia" state.
If intravascular hemolysis occurs, haptoglobin binding of hemoglobin $\alpha \beta$ dimers limits oxidative tissue damage from the release of free hemoglobin. This irreversible binding depletes haptoglobin from the circulation, and plasma levels take up to a week to recover. ${ }^{9}$ Thus, low serum haptoglobin represents not only a marker of hemolysis, but also an index of potentially limited physiological compensation mechanisms. Serum haptoglobin was therefore added to standard care pathways for 27 HHT patients with potentially out-of-proportion iron requirements, identified from the 90 HHT patients reviewed June 2017-March 2018. One case is illustrated in Figure 2A. Demographic details of all 27 patients are presented in Online Supplementary Table S1. The patients were aged 19-81 years, and all were experiencing significant hemorrhage: fifteen had nosebleeds at least daily, and these could be arterial rate bleeds, lasting more than an hour. Epistaxis severity scores ${ }^{1}$ ranged from 0 to 8.59 out of 10 .

As detailed below, however, there was also evidence to suggest shortened intravascular red cell survival in 12 of the selected 27 cases, all of whom had anemia that had been solely ascribed to hemorrhage-induced iron deficiency. All were receiving supplementary iron, with five receiving intravenous iron at least monthly. None had a known hemoglobinopathy or red cell disorder.

- Haptoglobin was below the normal range in 7/27 cases (Figure 2B). Low values could not be explained by impaired liver synthetic function as judged by serum 
Table 1. Haptoglobin relationships in 18 HHT patients who had NOT received a blood transfusion in the previous month.

\begin{tabular}{|c|c|c|c|c|c|}
\hline & & Crude & sion & CRP-adj] & ssion \\
\hline & Range (median) & coefficient & P & coefficient & P \\
\hline Haptoglobin (g/L) & $0-3.47(1.1)$ & - & - & - & - \\
\hline C-reactive protein (mg/L) & $0.3-14.3(2.5)$ & 0.11 & 0.023 & - & - \\
\hline Red blood cells (x10 $/ \mathrm{L})$ & $3.27-5.83(4.61)$ & 0.74 & 0.005 & 0.84 & $<0.001$ \\
\hline Hematocrit & $0.29-0.44(0.40)$ & 7.91 & 0.069 & 10.5 & 0.006 \\
\hline $\mathrm{MCV}(\mathrm{fl})$ & $71.7-95.8(87.8)$ & -0.065 & 0.018 & -0.06 & 0.014 \\
\hline Lymphocyte count $\left(x 10^{9} / \mathrm{L}\right)$ & $1-3(1.6)$ & 0.59 & 0.13 & 0.84 & 0.015 \\
\hline Hemoglobin $(\mathrm{g} / \mathrm{L})$ & $88-147(131.5)$ & 0.011 & 0.32 & 0.025 & 0.023 \\
\hline White blood cells $\left(\times 10^{9} / \mathrm{L}\right)$ & $3.5-8.6(7.2)$ & 0.31 & 0.012 & 0.27 & 0.027 \\
\hline APTT $(s)^{\dagger}$ & $20.4-29.9(24.5)$ & -0.13 & 0.048 & -0.12 & 0.041 \\
\hline Epistaxis severity score & $0-8.59(6.23)$ & -0.073 & 0.24 & -0.113 & 0.045 \\
\hline Reticulocytes $(\%)^{\ddagger}$ & $0-5.3(1.55)$ & -0.089 & 0.57 & -0.30 & 0.047 \\
\hline $\mathrm{MCH}$ & 22.3-30.7 (28.5) & -0.16 & 0.015 & -0.13 & 0.054 \\
\hline Basophil count $\left(\mathrm{x} 10^{9} / \mathrm{L}\right)$ & $0-0.1(0)$ & -8.67 & 0.08 & -8.30 & 0.07 \\
\hline Albumin & $27-46(39)$ & 0.013 & 0.76 & 0.078 & 0.075 \\
\hline Intravenous iron & Once - weekly (8-12 weekly) & -0.15 & 0.20 & -0.17 & 0.10 \\
\hline Fibrinogen & $1.86-5.40(3.14)$ & 0.55 & 0.11 & 0.44 & 0.11 \\
\hline Prothrombin time (s) & $10.2-12.7(11.2)$ & -0.43 & 0.22 & -0.49 & 0.12 \\
\hline Reticulocyte count & $0-174.4(71.1)$ & -0.0002 & 0.97 & -0.007 & 0.16 \\
\hline
\end{tabular}

$\mathrm{N}=18$ except for ${ }^{\dagger} \mathrm{N}=17$, and ${ }^{*} \mathrm{~N}=10$. Regression analyses were performed with $\ln$ (haptoglobin) as this was more normally distributed than haptoglobin (data not shown) Further relationships where adjusted $P$-values exceed 0.16 are provided in Online Supplementary Table S2- this includes sex, bilirubin, and red cell distribution width ( $P$-values 0.38-0.93). CRP: C-reactive protein; MCV: mean corpuscular volume; $\mathrm{MCH}$ : mean corpuscular haemoglobin; APTT: activated partial thromboplastin time

albumin, and some haptoglobin values may have been elevated as an acute phase response due to subtle confounding inflammation.

- Of the eleven manually-evaluated blood films performed at the time of haptoglobin measurements, polychromasia was observed in $8 / 11(72.7 \%)$, and spherocytes or elliptocytes in $5 / 11(45.5 \%)$. These findings were seen as commonly as consequences of iron deficiency (Figure 2C).

- The process appeared more indolent than immunemediated hemolysis: none of the eleven tested lactase dehydrogenase values exceeded $280 \mathrm{U} / \mathrm{L}$, and bilirubin was $\leq 12 \mathrm{mmol} / \mathrm{L}$ in $21 / 24$ cases. To date, all direct antibody and hemoglobin electrophoresis tests have been negative (including in 4 patients with haptoglobin $<0.5 \mathrm{~g} / \mathrm{L})$. However, adding to the index case hemoglobinopathy traits, a hemolytic risk factor was subsequenty identified in two of the haptoglobin cohort: glucose-6phosphate dehydrogenase (G6PD) deficiency in one, ${ }^{9}$ and severe hypophosphatemia ${ }^{10}$ in a second. In a third with recurrently subnormal serum haptoglobin, there was an intriguing spontaneous hemoglobin increment of $34 \mathrm{~g} / \mathrm{L}$ (without any change in nosebleeds) after an incidental 7day course of prednisolone $30 \mathrm{mg}$ od for asthma (Figure 2A). The magnitude and response duration exceeded that from four different erythrocyte transfusions in the preceding year.

Since recently-transfused red blood cells have shorter circulatory lifespans, ${ }^{9}$ analyses concentrated on the 18 patients who had not been transfused in the previous month. Crude regression analyses demonstrated that HHT patients with lower haptoglobin had fewer erythrocytes $(P<0.001)$, lower hematocrit $(P=0.006)$ and lower hemoglobin $(P=0.023)$ indicative of more severe anemia (Table 1). Findings were confirmed after adjustment for markers of inflammation using C-reactive protein (CRP,
Table 1). CRP-adjusted regression also indicated trends for lower haptoglobin to be associated with a higher percentage of reticulocytes $(P=0.047)$, higher absolute reticulocyte counts $(P=0.16)$, and greater use of intravenous iron $(P=0.10$, Table 1$)$. A striking feature was that patients with sub-normal haptoglobin did not demonstrate usual iron deficient erythrocyte indices but tended to have normal MCV (Figure 2D) and $\mathrm{MCH}$, even when anemic or iron deficient.

PubMed searches for "hemolysis" and "HHT" identified no publications on HHT, but it should not be a surprise that erythrocytes may have shorter life-spans in HHT patients with disturbed blood flow through arteriovenous malformations. Turbulent flow is a risk factor for hemolysis in the general population. ${ }^{9}$ Even the smallest HHT telangiectasia usually contain direct arteriovenous communications, ${ }^{11}$ larger pulmonary and hepatic AVMs each affect approximately $50 \%$ of cases, ${ }^{4}$ and HHT patients have higher than normal cardiac outputs to compensate for AVMs. ${ }^{12}$ Intravascular hemolysis occurs when the burden of erythrocyte membrane injury exceeds erythrocytic repair capacity. ${ }^{9}$ One aspect to consider is whether transient rises in serum iron and TfSI injure red cells as seen for endothelial cells. ${ }^{6,13}$ It is noteworthy that erythrocyte survival was normal when measured in non HHT patients with $\mathrm{Hb} \mathrm{S}^{14}$ trait at sea level. Similarly, where hemolysis was identified in $\mathrm{Hb} \mathrm{D}$ Punjab trait, it was attributed to coexisting hemoglobinopathies. ${ }^{15}$ We speculate that added stresses of the HHT vasculature, possibly combined with mild thrombotic angiography due to endothelial cell damage, may serve as stressors to normal erythrocytes, or erythrocytes with otherwise subclinical abnormalities, resulting in their indolent but clinically significant early destruction. Hence reduced in vitro HHT erythrocyte deformability is predicted.

In conclusion, shortened intravascular erythrocyte sur- 
vival should be considered as a potential contributor to anemia in HHT if iron requirements are out-of-proportion to hemorrhagic losses. Erythrocyte protection strategies may then prove helpful adjunctive treatments and represent an important focus for future studies.

Lieze Thielemans, ${ }^{1}$ D. Mark Layton ${ }^{2}$ and Claire L. Shovlin ${ }^{3}$

Imperial College School of Medicine, Imperial College London; ${ }^{2}$ Haematology, Imperial College London and ${ }^{3}$ NHLI Vascular Sciences, Imperial College London, UK

Acknowledgments: MB and CLS acknowledge support from the NIHR Biomedical Research Centre Funding Scheme (Imperial BRC). Ethical approved was from the Hammersmith, Queen Charlotte's, Chelsea, and Acton Hospital Research Ethics Committee, LREC 2000/5764: Case Notes Review: Hammersmith Hospital patients with pulmonary arteriovenous malformations and hereditary haemorrhagic telangiectasia (HHT). The authors would like to thank colleagues and former students in the VASCERN HHT Reference Network Centre at Hammersmith Hospital, Imperial College Healthcare NHS Trust. CLS also acknowledges support from the National Institute of Health Research (NIHR) Biomedical Research Centre Funding Scheme (Imperial BRC); National Institute of Health Research London (NW) Comprehensive Local Research Network; HHT patient donations; and Imperial College BSc project funds (for LT). The funders played no role in the design or conduct of the study; collection, management, analysis, or interpretation of the data; or preparation, review, or approval of the manuscript.

Correspondence:c.shovlin@imperial.ac.uk doi:10.3324/haematol.2018.205682

Information on authorship, contributions, and financial \& other disclosures was provided by the authors and is available with the online version of this article at www. haematologica.org.

\section{References}

1. Hoag JB, Terry P, Mitchell S, Reh D, Merlo CA. An epistaxis severity score for hereditary hemorrhagic telangiectasia. Laryngoscope. 2010;120(4):838-843.
2. Kjeldsen A, Kjeldsen J. Gastrointestinal bleeding in patients with hereditary hemorrhagic telangiectasia. Am J Gastroenterol. 2000;95(2):415-418.

3. Lund VJ, Darby Y, Rimmer J, et al. Nasal closure for severe hereditary haemorrhagic telangiectasia in 100 patients. The Lund modification of the Young's procedure: a 22-year experience. Rhinology. 2017;55(2):135-141.

4. Shovlin CL, Buscarini E, Kjeldsen AD, et al. European Reference Network for Rare Vascular Diseases (VASCERN) outcome measures for hereditary haemorrhagic telangiectasia (HHT). Orphanet J Rare Dis. 2018;15;13(1):136.

5. Finnamore $\mathrm{H}$, Le Couteur J, Hickson $\mathrm{M}$, et al. Hemorrhage-adjusted iron requirements, hematinics and hepcidin define hereditary hemorrhagic telangiectasia as a model of hemorrhagic iron deficiency. PLoS One. 2013;8(10):e76516.

6. Shovlin CL, Gilson C, Busbridge M, et al. Can iron treatments aggravate epistaxis in some patients with hereditary hemorrhagic telangiectasia? Laryngoscope. 2016;126(11):2468-2474.

7. Boother EJ, Brownlow S, Jackson JE, Shovlin CL. Cerebral abscess associated with odontogenic bacteremias, hypoxemia, and iron loading in immunocompetent patients with right-to-left shunting through pulmonary arteriovenous malformations. Clin Infect Dis. 2017;65(4):595-603.

8. Lopez A, Cacoub P, Macdougall IC, Peyrin-Biroulet L. Iron deficiency anaemia. Lancet. 2016;387(10021):907-916.

9. Glader B. Destruction of erythrocytes. Chapter 8 pp156-169 in Wintrobe's Clinical Hematology 12th Edition. Eds Greer JP, Foerster J, Rodgers GM, Paraskevas F, Glader B, Arber DA, and Means RT Jr. Lippincort Williams \& Wilkins, Philadelphia, 2009.

10. Melvin JD, Watts RG. Severe hypophosphatemia: a rare cause of intravascular haemolysis. Am J Hematol. 2002;69(3):223-224.

11. Braverman IM, Keh A, Jacobson BS. Ultrastructure and three-dimensional organization of the telangiectases of hereditary hemorrhagic telangiectasia. J Invest Dermatol. 1990;95(4):422-427.

12. Shovlin CL. Circulatory contributors to the phenotype in hereditary hemorrhagic telangiectasia. Front Genet. 2015;6:101.

13. Mollet IG, Patel D, Govani FS, et al. Low dose iron treatments induce a DNA damage response in human endothelial cells within minutes. PLoS One. 2016;11(2):e0147990.

14. Barbedo MMR, McCurdy PR. Red cell life span in sickle cell trait. Acta Haematol. 1974;51(6):339-343.

15. Pandey S, Mani Mishra R, Pandey S, Shah V, Saxena R. Molecular characterization of hemoglobin D Punjab traits and clinical-hematological profile of the patients. Sao Paulo Med J. 2012;130(4):248-251 